\title{
Der Übergang Schule-Hochschule aus Sicht der AG cosh
}

\author{
Klaus Dürrschnabel
}

\begin{abstract}
Es ist erfreulich, dass dem Übergang Schule-Hochschule in den Mitteilungen aktuell ein seiner Bedeutung entsprechender Stellenwert beigemessen wird. In der letzten Ausgabe wurden diesem Thema viele Seiten gewidmet und die Einschätzungen wichtiger Repräsentanten der Mathematikausbildung eingeholt. Um eine Vergleichbarkeit mit den Antworten der Interviews herzustellen, orientiert sich dieser Artikel an den dort gestellten Fragen, wobei wir uns erlaubt haben, manche Fragestellungen zu modifizieren bzw. zu ergänzen.
\end{abstract}

Woher kommt die Mathematik-Lücke zwischen Schule und Hochschule?

Der Unterricht an der Schule hat sich infolge der für Deutschland enttäuschenden Studien TIMSS und PISA verändert. Selbstentdeckendes Lernen, aus Fehlern lernen, Anwendungsbezug, Kompetenzorientierung und vieles mehr hielten nach der Jahrtausendwende Einzug, die Bedeutung des klassischen Frontalunterrichts ging zurück. Das musste notwendigerweise zu Lasten der fachlichen Inhalte gehen, zumal zusätzlich die Unterrichtsstunden in Mathematik in den vergangenen Jahrzehnten reduziert wurden (an Gymnasien in Baden-Württemberg etwa von bis zu 44 Wochenstunden zu Beginn der 8 oer Jahre auf einheitlich 32 Wochenstunden mit Einführung des G8) und gleichzeitig die Übergangsquoten aufs Gymnasium gestiegen sind.

Von diesen Veränderungen in den Schulen haben die Lehrenden an den Hochschulen nur partiell Notiz genommen. Sie setzen häufig nach wie vor die inhaltlichen Kenntnisse voraus, die vor 20, 30 oder noch mehr Jahren in der Schule vermittelt wurden. Leidtragende sind die Studienanfängerinnen und -anfänger, die den Schritt von der Schule zur Hochschule machen müssen mit der Konsequenz, dass unzureichende Mathematikvorkenntnisse häufig für einen Studienabbruch verantwortlich gemacht werden. ${ }^{1}$

Wie sollte der Mathematikunterricht in der Schule idealerweise verändert werden?

Die meisten Lehrerinnen und Lehrer in der Schule leisten im Rahmen ihrer Möglichkeiten hervorragende Arbeit. Leider sind die Rahmenbedingungen nicht so, wie sie für eine angemessene Vorbereitung auf ein Studium insbesondere im WiMINT-Bereich notwendig sind. Dazu wäre eine Erhöhung der Stundenzahl in Mathematik notwendig, um von der Hochschule geforderte Inhalte wie Binomische
Formeln, Termumformungen, Wurzel- und Betragsgleichungen, Ungleichungen oder komplexere Differenziationsregeln und Vektorgeometrie wieder behandeln und insbesondere auch üben zu können. Gegebenenfalls ist in der Sekundarstufe 2 die Trennung in Grund- und Leistungskurse sinnvoll, um für alle eine solide Allgemeinbildung in den Grundkursen zu gewährleisten und in den Leistungskursen eine adäquate Vorbereitung auf ein WiMINT-Studium für Interessierte sicherzustellen.

Die IQB-Bildungsstandards verstehen sich als Regelstandards, die angeben, welches Kompetenzniveau Schülerinnen und Schüler im Durchschnitt in einem Fach erreichen sollen. Als bundesweite Normierung der schulischen Mathematikausbildung sind sie sinnvoll, sie bilden eine Basis, auf der die Bemühungen zur Schließung der Lücke aufbauen können. Leider sind die Bildungsstandards an vielen Stellen unpräzise. Sie bedürfen einer „Nachschärfung", also einer Konkretisierung, wobei die Einbeziehung von Schulpraktikern und Hochschullehrenden aus unserer Sicht notwendig ist. Kritisch wird es, wenn - wie teilweise formuliert - die Bildungsstandards als absolut fixe Rahmenvorgabe angesehen werden, der sich alle Bemühungen zur Schließung der Lücke unterzuordnen haben.

Wie sollte die Mathematikvorlesung in der Hochschule idealerweise verändert werden?

Die meisten Mathematikvorlesungen werden von den Studierenden als gut bis sehr gut beurteilt, das belegen die Evaluationen der Hochschulen. Bei einem Misserfolg suchen die Studierenden häufig die Schuld bei sich beziehungsweise in der mangelnden Vorbereitung seitens der Schule, wobei die wahren Gründe oft in der nicht abgestimmten Vermittlungsweise zwischen Schule und Hochschule sowie in den nicht mehr in der Schule vermittelten, aber an der Hochschule vorausgesetzten Inhalten zu suchen sind. Der zunehmende Mangel an Selbstorgani- 


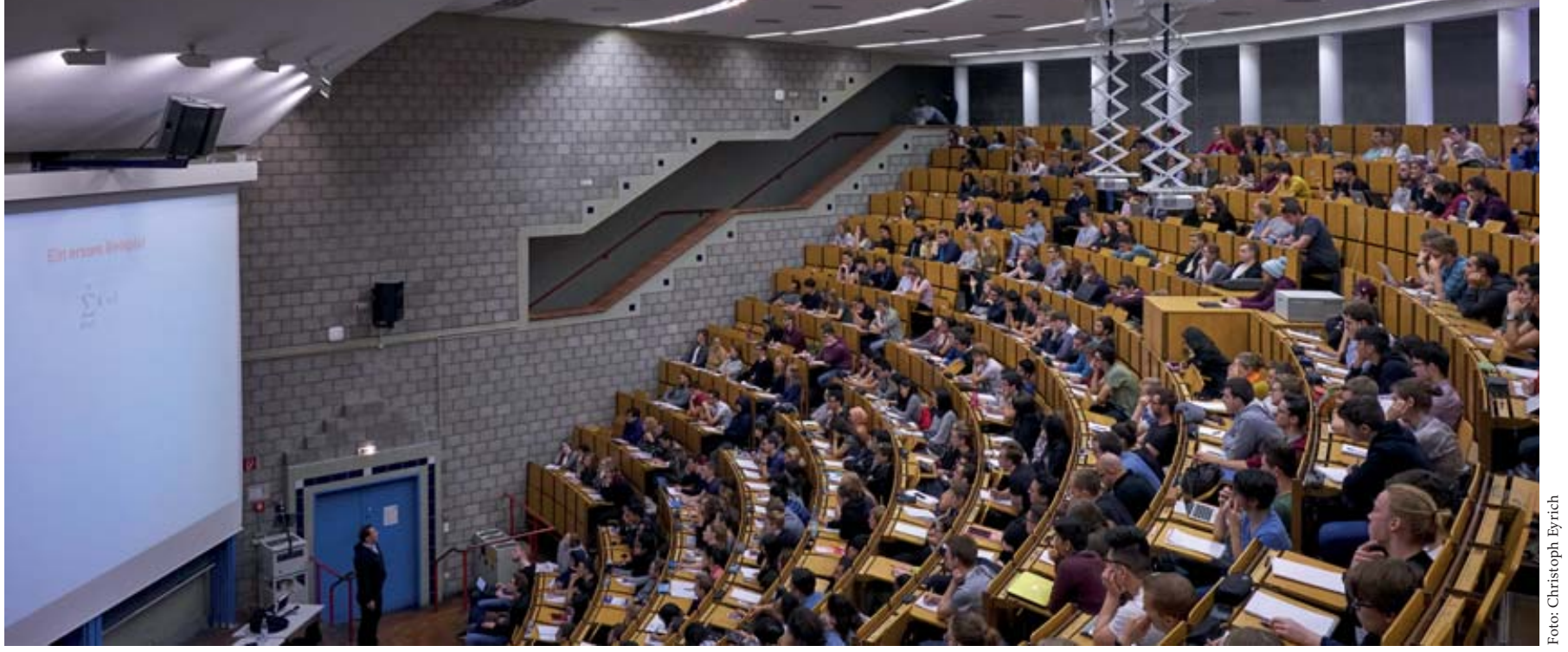

Semesterbeginn: Computerorientierte Mathematik I, Technische Universität Berlin

sation seitens der Studierenden aufgrund neuer Freiheiten an der Hochschule verstärkt diese Effekte.

Gerade Studierende der Wirtschaftswissenschaften oder des Ingenieurwesens, die Mathematik als Hilfswissenschaft benötigen, tun sich mit den klassischen Mathematikvorlesungen zunehmend schwer, eine Fachdidaktik für diese Klientel existiert nur in Ansätzen. Die viel diskutierten Methoden der Hochschuldidaktik wie „Inverted Classroom“ oder "Just in Time Teaching“ lösen gerade in Mathematik das Problem aufgrund ihres radikalen Bruchs mit der Schulausbildung nur teilweise. Von daher wäre die Entwicklung einer Fachdidaktik Mathematik für WiMINT unter Einbeziehung der Erfahrungen der Schulen mit ihren geänderten Lehrmethoden dringend erforderlich.

\section{Welche konkreten Schritte sind vordringlich?}

Höchste Priorität hat eine intensive Kommunikation zwischen Lehrenden an Schulen und Hochschulen, um sich der Veränderungen auf der jeweils anderen Seite bewusst zu werden und darauf reagieren zu können. Genau zu diesem Zweck hat sich in Baden-Württemberg im Jahr 2002 die AG cosh (Cooperation Schule Hochschule) gebildet, die sich die Glättung des Übergangs von der Schule zur Hochschule im Bereich Mathematik zur Aufgabe gemacht und unter anderem den viel zitierten Mindestanforderungskatalog Mathematik als Beschreibung des gewünschten Niveaus für die Schnittstelle zu einem WiMINT-Studium vorgelegt hat.

Ebenso ist die bundesweite Mathematik-Kommission Übergang Schule-Hochschule der Mathematik-Verbände DMV, GDM und MNU eine solche Plattform, um den Austausch zwischen Schule und Hochschule unter Einbeziehung der zuständigen Ministerien zu fördern. Die Tagung in Münster war ein mustergültiges Ergebnis eines gelungenen Informations- und Meinungsaustauschs. Wichtig ist auch eine regionale Kommunikation zwischen
Schulen und Hochschulen, um vor Ort einen gegenseitigen Informationsaustausch zwischen den Lehrenden an Schule und Hochschule, aber auch zwischen Schülern und Studierenden sicherzustellen. Diese regionalen Kooperationen werden zum Beispiel in Baden-Württemberg gerade aufgebaut.

Trotz aller genannten Bemühungen wird sich die Lücke zwischen Schule und Hochschule ohne die genannte Erhöhung der Stundenzahl in Mathematik nicht wesentlich verkleinern lassen.

\section{Welche Rolle spielt die Kompetenzorientierung?}

Der Begriff Kompetenzorientierung wird häufig kontrovers diskutiert, weil er einseitig dafür verantwortlich gemacht wird, dass ehemals verbindliche Inhalte in der Schule nicht mehr vermittelt werden. Wenn zugunsten der reinen Kompetenzorientierung die Inhalte zu stark zurückgedrängt, vielleicht sogar komplett ausgeblendet werden, ist diese Kritik durchaus gerechtfertigt. Wenn aber die fachlichen Inhalte eine angemessene Rolle spielen, ist die Kompetenzorientierung in Schule und Hochschule ein Fortschritt, schließlich geht es gerade im Bereich WiMINT darum, mathematisch zu argumentieren und mathematische Methoden auf neue Problemstellungen anzuwenden. Sicherlich ist es falsch, einem missverstandenen Kompetenzbegriff alleine die Schuld an der größer werdenden Lücke zwischen Schule und Hochschule zuzuschreiben.

Fast jeder hat eine Meinung zur Lehre in Mathematik. Welche Tatsachen werden gerne übersehen?

Die wachsende Lücke zwischen Schule und Hochschule hat multikausale Ursachen und ist nicht, wie manchmal behauptet, durch eine einzige Maßnahme zu schließen. So nimmt die Anzahl der Studienanfängerinnen und Studienanfänger $\mathrm{zu}$, inzwischen nehmen mehr als $50 \%$ eines 
Jahrgangs ein Studium auf. Die größer werdenden Studierendengruppen und die zunehmende Heterogenität erschweren zudem den Studieneinstieg. Die reduzierte Stundenzahl im Schulbereich in Verbindung mit häufigem Stundenausfall und einer Verkürzung der Gymnasialzeit, trägt ebenfalls zur Vergrößerung der Lücke bei. Studienanfängerinnen und -anfänger mit Fachhochschulreife haben aufgrund der nochmals reduzierten Mathematikstunden noch schlechtere Startvoraussetzungen.

Auch im Hochschulbereich wurden mit der Umstellung auf die Bologna-konformen Bachelorstudiengänge die Semesterwochenstunden in Mathematik vielfach gekürzt und die notwendigen Übungsphasen in den Selbstlernbereich ausgelagert. Von Hochschullehrenden wird häufig der unterschiedliche Bildungsauftrag von Schule (allgemeinbildende Mathematik) und Hochschule (fachbezogene Mathematik) übersehen. Zudem hat sich der soziale Kontext der Studierenden in den vergangenen Jahren verändert: So sind Studierende heute meist jünger, vermehrt Einzelkinder und die digitalen Medien mit allzeitiger Verfügbarkeit auch während der Lehrveranstaltungen haben selbstverständlich einen Einfluss auf sie.

\section{Welche konkreten Empfehlungen hat die AG cosh für die Schnittstelle Schule-Hochschule?}

Basierend auf den vorhergehenden Abschnitten lassen sich folgende Empfehlungen formulieren:

- Ohne eine direkte Kommunikation zwischen Schule und Hochschule ist ein reibungsfreier Übergang von vornherein zum Scheitern verurteilt. Schule und Hochschule müssen sich auf ein gewünschtes Niveau verständigen, auf das die Schulen hinarbeiten und auf dem die Hochschulen aufbauen. Die Jugendlichen müssen von der Schule auf den Übergang vorbereitet und von der Hochschule ohne überzogene Anforderungen an dieser definierten Schnittstelle abgeholt werden. Gegenseitige Schuldzuweisungen helfen den Studierenden wenig.

- Die Politik muss die Kommunikation zwischen Schule und Hochschule fördern und gemeinsamen Vorschlägen zur Realisierung verhelfen. Sie muss Rahmenbedingungen schaffen, die es Schulabgängerinnen und -abgängern ermöglichen, den Studieneinstieg gerade in Mathematik zu meistern. Gemeinsame Anforderungskataloge, Studienmodelle individueller Geschwindigkeiten sowie sonstige Hilfestellungen wie Lernzentren, Tutorien und Ähnliches sind dabei sicher hilfreich.

- Die aktuellen Stundenzahlen in Mathematik müssen erhöht werden, um grundlegende mathematische Fähigkeiten gerade aus der Sekundarstufe 1 fundiert unterrichten und üben zu können. Nur mit sicheren mathematischen Grundkenntnissen können die mathematischen Anforderungen in einem WiMINT-Studienfach gemeistert werden.

- Studienanfängerinnen und -anfänger mit Fachhochschulreife, die nicht über den klassischen gymnasialen Weg an eine Hochschule kommen, haben zu Studienbeginn mit den größten mathematischen Defiziten zu kämpfen. Andererseits schließen diese Studierenden nach Überwindung der Anfangsprobleme in Mathematik ihr Studium häufig mit überdurchschnittlichem Erfolg ab. Diesen Studierenden muss von Schule und Hochschule eine besondere Beachtung geschenkt werden.

- Die mathematischen Anforderungen an ein WiMINTStudium müssen den Studieninteressierten bereits vor Studienbeginn klar gemacht werden. Es macht keinen Sinn, diese herunterzuspielen oder mathematische Vorlesungen in Modulen mit wohlklingenden Namen zu verbergen. Der vielfache Studienabbruch ist die Folge.

- Die Schule hat große Erfahrungen im Feld der Fachdidaktik Mathematik, während im Hochschulbereich dieser Bereich für WiMINT-Studienfächer eher unterentwickelt ist. Die Expertise der Schulen in diesem Bereich sollte von den Hochschulen zur Entwicklung einer Fachdidaktik Mathematik für Wirtschafts- und Ingenieurstudierende genutzt werden.

Anmerkung

1. Vgl. z. B. Heublein u. a., Zwischen Studienerwartungen und Studienwirklichkeit, Ursachen des Studienabbruchs, beruflicher Verbleib der Studienabbrecherinnen und Studienabbrecher und Entwicklung der Studienabbruchquote an deutschen Hochschulen, DZHW Hannover 2017.

Prof. Dr. Klaus Dürrschnabel, Hochschule Karlsruhe - Technik und Wirtschaft, Moltkestraße 3o,76133 Karlsruhe. klaus.duerrschnabel@hs-karlsruhe.de

Klaus Dürrschnabel studierte Mathematik, Physik und Informatik an der Universität Karlsruhe. Nach seiner Dissertation im Bereich Differenzialgeometrie wechselte er 1992 in die freie Wirtschaft zu einem großen Lebensversicherer in Stuttgart. Seit 1996 ist er Professor für Mathematik und Informatik an der Hochschule Karlsruhe - Technik und Wirtschaft. Er ist Mitinitiator der AG cosh (Cooperation Schule Hochschule) in Baden-Württemberg, in der er bis heute im Kernteam mitarbeitet. 